\title{
Familienfreundlicher Betrieb - Einflussfaktoren aus Beschäftigtensicht
}

Mit der gewachsenen Erwerbsbeteiligung von Müttern und dem allmählich zunehmenden Engagement von Vätern in der Familie wird eine gelingende Vereinbarkeit von Familie und Beruf für immer mehr Menschen bedeutsam. Im Rahmen der Allianz für die Familie sollen in Deutschland endlich bessere Bedingungen dafür geschaffen werden, Beruf und Familie zu vereinbaren. Betriebe spielen hierbei eine wichtige Rolle. Sie können dieses Ziel durch familiengerechte Arbeitsbedingungen und spezielle Maßnahmen unterstützen. Welche Faktoren sind aus der Sicht von Arbeitnehmerinnen und Arbeitnehmern mit Kindern für die Vereinbarkeit von Familie und Beruf besonders wichtig? Dies wurde mit Hilfe multivariater Verfahren ermittelt.

\section{Problemstellung}

Die Bedingungen für die Vereinbarkeit von Familie und Beruf sind seit Langem in der Kritik. Bundesregierung und Vertreter von Gewerkschaften und Wirtschaft haben sich 2007 in einer gemeinsamen Erklärung „Deutschland braucht eine familienbewusste Arbeitswelt" auf neue Wege für mehr Familienfreundlichkeit verständigt (http:// www.bmfsfj.de/Politikbereiche/Familie/ familie-und-arbeitswelt,did=11408.html.).

Nur eine Minderheit von Betrieben hat bisher Maßnahmen zur besseren Vereinbarkeit von Familie und Beruf eingeführt (Möller/Allmendinger 2003), und nur ein kleiner Teil der Eltern kann auf solche Ansatzpunkte im Betrieb zurückgreifen (BMFSFJ 2004).

In den letzten Jahren sind besonders die betriebswirtschaftlichen und demografischen Wirkungen familienorientierter Maßnahmen betont worden. ${ }^{1}$ Als betriebswirtschaftliche Effekte werden reduzierte Fluktuation, geringere Fehlzeiten, erhöhte Produktivität und Einsparung von Wiedereingliederungs- und Personalbeschaffungskosten angesehen (Prognos 2003, 2004; Juncke 2005). Auch demografische und volkswirtschaftliche Effekte, wie steigende Geburtenraten und höhere Arbeitsmarktpartizipation von Frauen, werden erwartet (OECD 2001; BMFSFJ et al. 2004). Die hilfreiche Wirkung familienfreundlicher Maßnahmen für Eltern und Kinder wird dabei unterstellt. Aber wie wirken die Maßnahmen im Einzelnen tatsächlich? Welche Faktoren verbessern in welchem Maße die Vereinbarkeit von Familie und Beruf? Und wovon hängt es ab, ob die Beschäftigten ihren Betrieb als familienfreundlich ansehen? Dies ist bisher nicht geklärt.

Uns interessierten vor allem drei Fragen. Erstens: Welche Rolle spielen für die Einschätzung der Familienfreundlichkeit die unterschiedlichen Dimensionen der Arbeitszeit: ihre Dauer, ihre Lage sowie ihre Verteilung? Wie wirken sich flexible Arbeitszeitmodelle auf die Vereinbarkeit von Familie und Beruf aus? Zweitens, welche Bedeutung haben weitere arbeitsorganisatorische Faktoren sowie das Betriebsklima? Und drittens: Inwieweit verändern spezielle familienfreundliche betriebliche Maßnahmen die Bewertung?

Nur die Beschäftigten selbst können entscheiden, ob eine Maßnahme tatsächlich familienfreundlich ist und ihnen die Vereinbarkeit der Lebensbereiche erleichtert (OECD 2001, S. 147). Letztlich müssen sich die familienorientierten Maßnahmen in ihrem Arbeitsalltag bewähren. Zugleich müssen sie den Veränderungen der Geschlechterverhältnisse Rechnung tragen und auf die Gleichstellung von Frauen im Beruf und von Männern in der Familie ausgerichtet sein.

Wir haben mit Daten einer repräsentativen Befragung abhängig beschäftigter Mütter und Väter ${ }^{2}$ die Bewertung der Familienfreundlichkeit des Betriebes und die Einschätzung der Vereinbarkeit von Familie und Beruf mit multivariaten Verfahren untersucht. Im Abschnitt 2 werden der Forschungsstand und die zu prüfenden Hypothesen dargestellt. Im Abschnitt 3 werden die Methode und die wichtigsten Variablen der Befragung erläutert. Abschnitt 4 präsentiert die empirischen Ergebnisse. Im Abschnitt 5 werden Schlussfolgerungen gezogen.

\section{Bewertungsfaktoren der Vereinbarkeit}

\subsection{FORSCHUNGSSTAND}

Für die Vereinbarkeit von Familie und Beruf spielen Betriebe eine sehr wichtige Rolle, denn hier wird über einen Großteil der Bedingungen der beruflichen Tätigkeit entschieden (Dilger et al. 2007).

\footnotetext{
Vgl. Flüther-Hoffmann/Solbrig 2003; Prognos 2003, 2004; BMFSFJ/IW 2005; Juncke 2005. Eine auf die Bedürfnisse von Beschäftigten orientierte Sicht findet sich z. B. bei Habisch 1995, bezüglich der Arbeitszeiten u.a. bei Richter/Stackelbeck 1992; Büssing/Seifert 1995; Maschke et al. 2005. 2 Im Auftrag des BMFSFJ und des DGB wurde im November/Dezember 2003 vom WSI die Befragung "Erwartungen an einen familienfreundlichen Betrieb" in Kooperation mit TNS Emnid durchgeführt. Es wurden 2.000 abhängig beschäftigte Eltern mit Kind unter 18 Jahren sowie Beschäftigte mit regelmäßigen Pflegeaufgaben befragt (vgl. BMFSFJ 2004).
}

Christina Klenner, Dr., Wissenschaftlerin im
WSI in der Hans-Böckler-Stiftung.
Arbeitsschwerpunkte: Frauenerwerbstätig-
keit, Geschlechterverhältnisse, work-life
Balance.
e-mail: christina-klenner@boeckler.de
Tanja Schmidt, Dipl.-Soziologin, freiberuf-
liche Wissenschaftlerin (Schmidt-Sozial-
forschung). Arbeitsschwerpunkte:
Frauenerwerbstätigkeit, Erwerbs- und
Lebensverlaufsforschung.
e-mail: kontakt@schmidt-sozialforschung.de


Unter den betrieblichen Bedingungen sind Arbeitszeiten von entscheidender Bedeutung, da eine zeitliche Konkurrenz zwischen den Anforderungen aus beiden Lebenssphären besteht (Juncke 2005). Im Rahmen unserer Beschäftigtenbefragung (vgl. Fn 2) wurden familienfreundliche Arbeitszeiten als der mit Abstand wichtigste Bereich für einen familienfreundlichen Betrieb benannt.

Deskriptive Analysen sowie qualitative Untersuchungen haben gezeigt, dass vor allem kürzere Arbeitszeiten familienfreundlich sind. Je länger die tatsächliche Arbeitszeit, desto schlechter wird deren Vereinbarkeit mit dem Familienleben eingeschätzt (European Foundation 2003; BMFSFJ 2004; Bloom et al. 2006). Hinsichtlich der Arbeitszeitlage gilt Arbeit am Abend, in der Nacht oder am Wochenende als schwer vereinbar mit der Kinderversorgung (Rinderspacher 1987; La Valle et al. 2002). Die Auswirkungen von flexiblen Arbeitszeiten hängen von der konkreten Ausgestaltung ab: Es kommt vor allem darauf an, wer die Kontrolle über die Arbeitszeit ausübt (Bauer et al. 2004; Eberling et al. 2004; Munz 2006). Familiengerecht sind Arbeitszeiten dann, wenn sie von einer stabilen Grundstruktur ausgehend flexible Reaktionen auf familienbedingte Zeitbedarfe erlauben (Klenner/Pfahl 2005; Sachverständigenkommission 2005). Gleitzeitarbeit wird hierzu als förderlich angesehen (Bundesmann-Jansen et al. 2000), ebenso bezahlte Freistellung bei Erkrankung des Kindes. Auch die Möglichkeit, zwischen Teilzeitarbeit und Vollzeitarbeit zu wechseln sowie bestimmte Schichten zu wählen (Bond et al. 2003), verbessert die Vereinbarkeit von Familie und Beruf. Negativ wirken sich Arbeitszeiten aus, auf die die Beschäftigten wenig oder keinen Einfluss haben, sowie solche, die stark und unvorhersehbar schwanken und dadurch die Planbarkeit des Alltagslebens komplizieren (Seifert 2005).

Auch Faktoren der Arbeitsorganisation spielen für die Vereinbarkeit beider Sphären eine Rolle. Die Selbstbestimmung über Arbeitsabläufe (Thomas/Ganster 1995) kann die Einschätzung beeinflussen, ob der Betrieb familienfreundlich ist. Gleiches gilt für die Möglichkeit, von zu Hause in Form alternierender Telearbeit zu arbeiten (Bloom et al. 2006).

Weitere wichtige Faktoren sind das betriebliche Klima und das Verständnis der Vorgesetzten (Weber 2006; Saltzstein et al. 2001; Bond et al. 2003). Das betriebliche
Klima zeigt sich unter anderem daran, wie Kollegen und Vorgesetzte reagieren, wenn familienbezogene Rechte in Anspruch genommen werden und es dadurch zu Fehlzeiten kommt (Elternzeit, Freistellung wegen eines kranken Kindes).

Weitere Faktoren sind familienfreundliche betriebliche Maßnahmen. Von ihnen wird definitionsgemäß angenommen, dass sie sich positiv auf die Vereinbarkeit auswirken. Hierzu zählen beispielsweise Betriebskindergärten sowie das Angebot einer Notfallbetreuung für Kinder, wenn die normale Kinderbetreuung einmal ausfällt, außerdem familienbezogene Geldleistungen oder Vermittlungs- und Beratungsdienstleistungen. Solche Maßnahmen können Stress, Belastungen und gesundheitliche Beschwerden reduzieren (Thomas/ Ganster 1995) sowie die Arbeitszufriedenheit steigern (Saltzstein et al. 2001; Bond et al. 2003).

Unter den strukturellen Faktoren wird vor allem die Größe des Betriebes als beeinflussend angesehen: Größere Betriebe stellen mehr Maßnahmen zur Verfügung (Flüter-Hoffmann/Seyda 2006; Bloom et al. 2006). Auch der Anteil weiblicher Manager im Betrieb macht einen entscheidenden positiven Unterschied (ebd.).

Multivariate Analysen zum Thema gibt es nur vereinzelt. ${ }^{3}$ Eine solche Analyse auf Basis des dritten „European Working Conditions Survey“ 2000/2001 kam für Europa zum Ergebnis, dass vor allem lange Arbeitszeiten, Wegezeiten zur Arbeit und ,unsoziale Arbeitszeiten", besonders Abend-, Nachtarbeit und Samstagarbeit, den Konflikt zwischen Arbeit und Familienleben signifikant verschärfen. Niedrige Arbeitsintensität sowie der Grad an persönlicher Einflussmöglichkeit auf die Arbeitszeit wirken dagegen positiv. Daneben sind das Alter und die Familienform der Beschäftigten wichtige Einflussfaktoren (OECD 2004, S. 47).

\subsection{HYPOTHESEN}

Viele Faktoren beeinflussen die Einschätzung der Vereinbarkeit von Familie und Beruf (Abbildung 1). Neben persönlichen Merkmalen der Befragten sind es vor allem Bedingungen im Betrieb in ihrem Zusammenspiel mit gesetzlichen Regelungen (Mutterschutz, Elternzeit und finanzielle Familienleistungen), mit Regelungen in Tarifverträgen (Arbeitszeiten, Freistellungsrechte oder familienfreundliche Maßnahmen) und regionalen bzw. kommunalen Bedingungen (Infrastruktur an Kinderbetreuung, schulische Angebote sowie der öffentliche Nahverkehr).

Folgende Hypothesen über den Einfluss betrieblicher Faktoren ${ }^{4}$ auf die Bewertung der Vereinbarkeit von Familie und Beruf werden geprüft:

(1) Die Arbeitszeit beeinflusst die Bewertung in dominierender Weise:

- Je kürzer die Dauer der Arbeitszeit, umso besser wird die Vereinbarkeit von Familie und Beruf bewertet.

- Je häufiger Arbeit mit einer unsozialen Lage verrichtet wird - also am Abend, in der Nacht, am Samstag und am Sonntag desto schlechter wird die Vereinbarkeit beurteilt.

- Es wird angenommen, dass die Möglichkeiten, die eigene Arbeitszeit an familiäre Zeitbedarfe anzupassen, durch Gleitzeitarbeit, Überstundenkonten, Vertrauensarbeitszeit sowie Arbeitszeitabstimmung im Team erweitert werden und dadurch die Vereinbarkeit besser beurteilt wird.

- Je mehr die Arbeitszeit durch betrieblich veranlasste Flexibilität schwankt, umso geringer sind die Regelmäßigkeit und die Planbarkeit der Arbeitszeiten und desto schlechter ist die Vereinbarkeit. Hierzu können Drei-Schichtarbeit, Arbeitszeitkonten, die zur Erleichterung betrieblich veranlasster Arbeitszeitschwankungen eingeführt wurden, sowie Arbeit auf Abruf führen.

(2) Die Arbeitsorganisation beeinflusst die Beurteilung der Vereinbarkeit:

- Wenn Familienpflichten bei der Arbeitszeitfestlegung oder bei der Urlaubsplanung berücksichtigt werden und wenn die Arbeit teilweise von zu Hause aus möglich ist (Telearbeit), verbessert das die Vereinbarkeitsbewertung.

- Wechselnde Arbeitsorte oder Dienstreisen verschlechtern die Vereinbarkeit von Familie und Beruf.

\footnotetext{
3 Für eine multivariate Analyse für Deutschland vgl. Weber 2006. Hier wurden die Auswirkungen von familienfreundlichen Initiativen über die Messung freiwilliger Fluktuation vorgenommen, was nur ein sehr indirekter Indikator ist.

4 Wir beschränken die Betrachtung vor allem auf betriebliche Faktoren in ihrem Zusammenspiel mit persönlichen Faktoren. Dies ergibt sich aus dem für die Untersuchung genutzten Datensatz.
} 


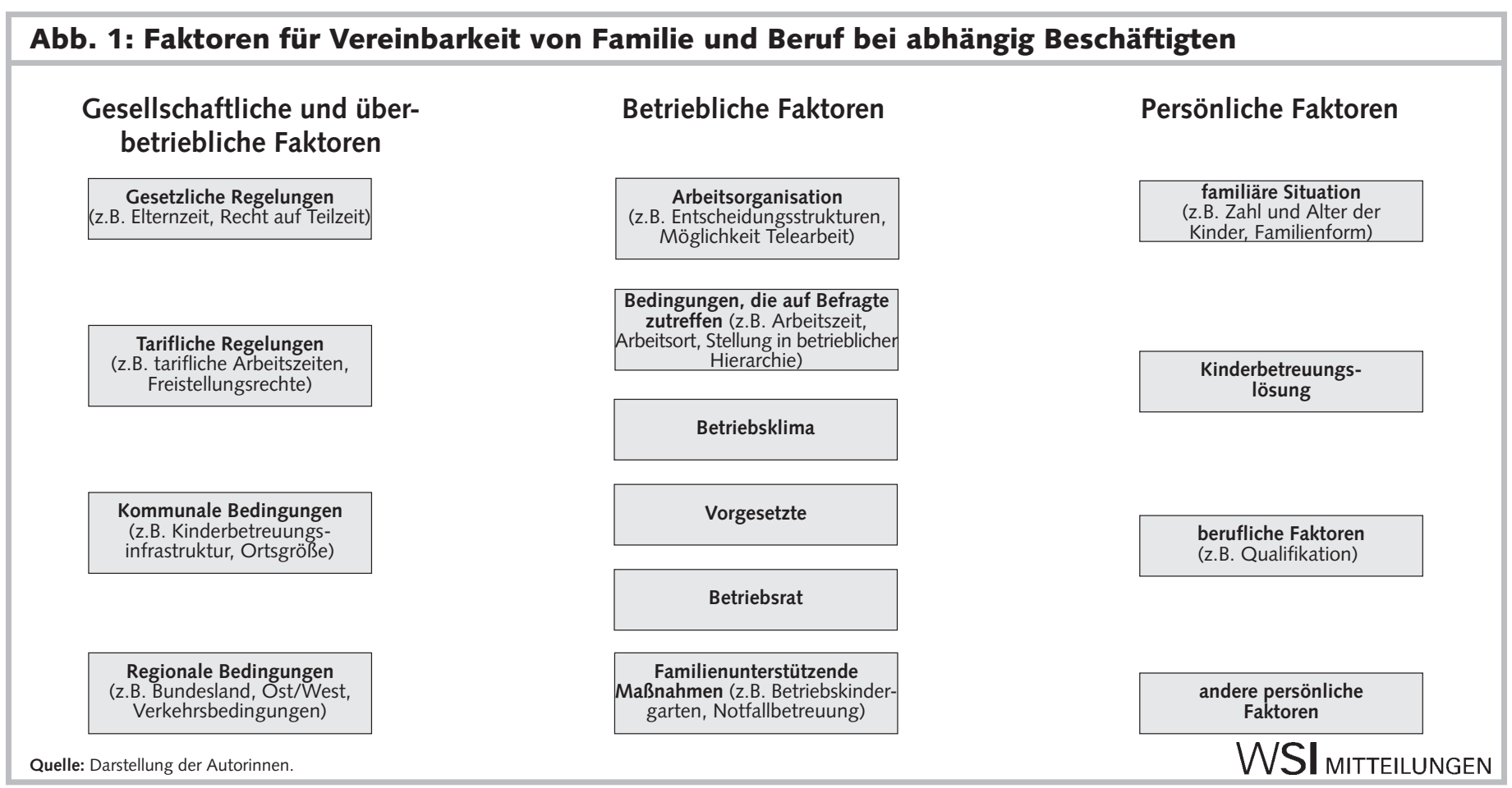

(3) Familienfreundliche betriebliche Maßnahmen verbessern die Bewertung der Vereinbarkeit von Familie und Beruf.

(4) Je größer die Zustimmung von Kolleginnen und Kollegen zur Inanspruchnahme familienbezogener Rechte und je geringer die ablehnende Reaktionen bei kindbedingten Fehlzeiten ist, desto besser ist die Vereinbarkeitsbewertung.

\section{Modell zur Schätzung der Vereinbarkeitsbewertung}

\subsection{DATEN}

Aus der Befragung „Erwartungen an einen familienfreundlichen Betrieb" liegen Informationen von 2.000 abhängig beschäftigten Eltern mit Kind unter 18 Jahren sowie mit Pflegeaufgaben vor. ${ }^{5}$ Für die hier dargestellte Analyse wurden nur Personen berücksichtigt, die mit mindestens einem Kind unter 18 Jahren oder mit einem behindertem Kind (ohne Altersbegrenzung) im eigenen Haushalt leben $(\mathrm{N}=1710)$.

\subsection{ABHÄNGIGE VARIABLEN}

Die Beurteilung der Vereinbarkeit von Beruf und Familie wird anhand zweier statistisch getrennter Themenbereiche gemes- sen: Das sind erstens, der Index zur Messung der Beurteilung der Vereinbarkeit von Arbeitszeit und Familie - „ArbeitszeitVereinbarkeits-Index" (MIXAZ) sowie die daraus komprimierte Variable „Gute Beurteilung der Vereinbarkeit von Arbeitszeit und Familie - Ja/Nein (AZGUT)“; zweitens, der Index zur Messung der Bewertung der Familienfreundlichkeit des Betriebes - „Familienfreundlichkeitsindex“ (MIXBBP) und die daraus komprimierte Variable „Gute Beurteilung der Familienfreundlichkeit des Betriebes - Ja/Nein“ (BTGUT). ${ }^{6}$

Zur Interpretation ist zu berücksichtigen, dass die Indizes konstruierte Maße der Zufriedenheit von Beschäftigten mit Kindern bezüglich der alltäglichen Bewältigung der widerstreitenden Anforderungen aus den Bereichen Beruf und Familie darstellen. Wenn man wissen will, was die Arbeitnehmerinnen und Arbeitnehmer selbst über eine mehr oder weniger gelingende Vereinbarkeit und über die Familienfreundlichkeit des Betriebes denken, sind Zufriedenheitswerte kaum durch objektive Maße ersetzbar.

\subsection{UNABHÄNGIGE VARIABLEN}

Zur Beschreibung und Erklärung von MIXAZ und AZGUT sowie von MIXBBP und BTGUT werden für die Modellbildung eine Reihe von unabhängigen Variablen herangezogen, von denen aufgrund vorlie- gender Forschungsergebnisse (Abschnitt 2) Einfluss vermutet wird.

In der ersten Gruppe von Variablen sind alle individuellen soziodemografischen Kontroll-Variablen wie Geschlecht, Anzahl der Kinder im Haushalt, Partner im Haushalt, Anzahl erwachsener Personen im Haushalt, Kinderbetreuungsnetzwerk oder berufliche Ausbildung der Befragten zusammengefasst. In der zweiten Gruppe sind Merkmale der beruflichen Tätigkeit, darunter Angaben zu Dauer und Lage der Arbeitszeiten, gebündelt. In der dritten Variablengruppe werden betriebliche Merkmale berücksichtigt, z. B. Arbeitszeitmodelle, Telearbeit, Bestimmung über die Arbeitsabläufe, Vorhandensein betrieblicher Infrastruktur an familienfreundlichen Maßnahmen sowie weitere Strukturmerkmale als Kontrollvariablen. In der vierten Gruppe sind zusätzliche betriebliche (Klima-)Merkmale abgebildet, vor allem Reaktionen von Kolleginnen und Kollegen auf kindbedingte Fehlzeiten.

$\mathrm{Zu}$ beachten ist, dass im Rahmen der Befragung betriebliche und persönliche,

5 Vgl. Fußnote 2 sowie ausführlich zur Stichprobe BMFSFJ 2004

6 Die abhängigen Variablen wurden mit Hilfe von umfangreichen Faktorenanalysen validiert. Das detaillierte Verfahren sowie alle hier nicht dargestellten bivariaten Ergebnisse und nicht signifikanten Variablen sind in der ausführlichen Fassung der Studie nachzulesen. 
jedoch nur wenige strukturelle Faktoren erhoben wurden. Kommunale Bedingungen oder tarifvertragliche Regelungen müssen daher ausgeklammert bleiben.

\section{Befunde}

\subsection{BIVARIATE ERGEBNISSE}

Zur bivariaten Überprüfung der Hypothesen führten wir Mittelwertvergleiche und Korrelationsanalysen durch. Es zeigt sich, dass nahezu alle Hypothesen durch signifikante Ergebnisse unterstützt werden. Kürzere Arbeitszeiten, familienbezogen flexible Arbeitszeiten sowie eine Arbeitsorganisation, die Familienbelange berücksichtigt, weisen einen positiven Zusammenhang zur Einschätzung der Vereinbarkeit von Beruf und Familie auf. Dagegen stehen Arbeitszeiten außerhalb der Normalarbeitszeit, Arbeitszeitschwankungen und wechselnde Arbeitsorte im negativen Zusammenhang zur Vereinbarkeitsbewertung.

Wenn flexible Arbeitszeitmodelle vorhanden sind, lassen sich eher positive $\mathrm{Zu}$ sammenhänge zur Vereinbarkeitsbewertung feststellen. Diese gelten für Überstundenkonten, Gleitzeitarbeit, Arbeitszeitabstimmung im Team, jedoch nicht für andere Arbeitszeitkonten zur Steuerung von Arbeitszeitschwankungen und Vertrauensarbeit. Günstig wirken ebenfalls familienunterstützende betriebliche Maßnahmen sowie verständnisvolle Kollegen. Auch die häusliche Situation ist von Bedeutung. Je älter das jüngste Kind ist, desto besser wird die Vereinbarkeit beurteilt. Ebenfalls positiv scheint sich eine partnerschaftliche Aufteilung der Familienarbeit zwischen den Eltern auszuwirken.

Doch welche Variablen haben den größten Einfluss? Und bleiben alle bivariaten Zusammenhänge bestehen, wenn alle unabhängigen Variablen gemeinsam - also auch im Hinblick auf ihre gegenseitige Abhängigkeit - berücksichtigt werden? Diese Fragen werden mit Hilfe einer multivariaten logistischen Regression auf die Wahrscheinlichkeit einer „Guten Beurteilung der Vereinbarkeit von Arbeitszeit und Familie“ (AZGUT) und auf die „Gute Bewertung der Familienfreundlichkeit des Betriebes" (BTGUT) analysiert.

\subsection{MULTIVARIATE ANALYSE}

Logistische Regressionen finden bei binären abhängigen Variablen Anwendung. Anstelle der üblicherweise geschätzten Koeffizienten geben wir marginale Effekte an, denn diese lassen eine unmittelbare Interpretation der Wirkungsrichtung und -höhe zu. Ermittelt werden die marginalen Effekte für eine Variable, indem die übrigen Variablen zu ihrem jeweiligen Mittelwert evaluiert sind (Long/Freese 2006). ${ }^{7}$

\section{GUTE BEURTEILUNG DER VEREINBARKEIT VON ARBEITSZEIT UND FAMILIE (AZGUT)}

Die signifikanten marginalen Effekte der logistischen Regression auf die gute Beurteilung der Vereinbarkeit von Arbeitszeit und Familie (AZGUT) gehen aus Tabelle 1 hervor. ${ }^{8}$

Der Einfluss der Familienform ist bemerkenswert. Den größten Einfluss auf die Wahrscheinlichkeit einer guten Beurteilung der Vereinbarkeit von Arbeitszeit und Familie hat die Frage, ob Eltern alleinerziehend sind oder in Paarhaushalten leben. ${ }^{9}$ Beschäftigte mit Partner haben eine um 22,3\% höhere Chance, die Vereinbarkeit gut zu bewerten als allein mit ihren Kindern Lebende (unter der Voraussetzung, dass alle anderen Merkmale der „mittleren Person" entsprechen). Alleinerziehende haben also grundsätzlich geringere Chancen, die Vereinbarkeit der Arbeitszeit mit dem Familienleben als gut einzuschätzen.

Die stärksten signifikanten Effekte auf die Chance einer guten Vereinbarkeitsbewertung haben darüber hinaus folgende Variablen:

- Die Dauer der tatsächliche Arbeitszeit: Je kürzer sie ist, umso größer ist die Wahrscheinlichkeit einer guten Bewertung der Vereinbarkeit. Bei den Alleinerziehenden ist dies die Variable mit dem größten Einfluss. - Wochenendarbeit: Beschäftigte, die immer oder häufig am Wochenende arbeiten müssen, haben hochsignifikant geringere Wahrscheinlichkeiten, die Vereinbarkeit von Arbeitszeit und Familie gut einzuschätzen, als solche, die nicht am Wochenende arbeiten müssen.

- Arbeit zu unvorhergesehenen Zeiten und Arbeit auf Abruf: Es wirkt sich ungünstig auf die Einschätzung der Vereinbarkeit aus, wenn die Arbeitszeiten kaum vorhersehbar und somit nicht planbar sind.
Großen Einfluss hat der arbeitsorganisatorische Umgang mit familiären Belangen im Betrieb. Werden diese bei der Arbeitszeitfestlegung berücksichtigt, schätzen die Befragten die Vereinbarkeit signifikant besser ein. Förderlich wirkt sich ebenfalls Telearbeit aus. Insoweit werden die bivariaten Ergebnisse bestätigt.

Überraschend ist dagegen, dass flexible Arbeitszeitmodelle, von denen allgemein angenommen wird, sie könnten auch für familiäre Zwecke genutzt werden, keine signifikanten Effekte auf die Beurteilung der Vereinbarkeit von Arbeitszeit und Familie haben. Das gilt für Gleitzeitarbeit und Überstundenkonten ebenso wie für Arbeitszeitabstimmung im Team oder Vertrauensarbeitszeit. Signifikant negativ wirken sich „andere Arbeitszeiten“ - das sind Nicht-Gleitzeit- oder Überstundenkonten - aus. Es zeigt sich, dass flexible Arbeitszeiten in der Bedeutung gegenüber Dauer und Lage sowie Planbarkeit der Arbeitszeiten eindeutig zurücktreten. Bemerkenswert ist ebenfalls, dass Nachtarbeit entgegen der Annahme keinen negativen Effekt hat.

Ebenfalls bedenkenswert ist die Tatsache, dass familienfreundliche Maßnahmen, die die Vereinbarkeit von Familie und Beruf erleichtern sollen, sich nicht auf die Wahrscheinlichkeit einer guten Bewertung der Vereinbarkeit von Arbeitszeiten und Familie auswirken. Dagegen sind zwei Faktoren von Einfluss, die bisher wenig beachtet werden:

(1) Die Kinderbetreuungslösung: Ein großes Betreuungsnetzwerk mit vielen Beteiligten geht mit einer deutlich geringeren Wahrscheinlichkeit der guten Bewertung der Vereinbarkeit von Arbeitszeit und Familie gegenüber anderen Betreuungslösungen einher. Hierfür könnte der höhere Koordinierungsaufwand verantwortlich sein.

(2) Die Größe des Wohnortes: Befragte, und hier wieder insbesondere Alleinerziehende, die in kleinen Orten mit 2.000 bis 5.000

7 An dieser Stelle bedanken wir uns herzlich be Holger Alda, SOFI Göttingen, für seine statistische Beratung.

8 Im Folgenden werden aus Platzgründen nur die signifikanten Effekte dargestellt und interpretiert.

9 Durch entsprechende Tests wurde sichergestellt, dass es sich hierbei nicht um getrennte Stichproben handelt. 
Tabelle 1: Logistische Regression - Abhängige Variable AZGUT (nur signifikante Koeffizienten)

\begin{tabular}{|c|c|c|c|c|c|c|c|c|c|c|}
\hline & Modell 1 & & & Modell 2 & & & Modell 3 & & & \\
\hline $\begin{array}{l}\text { Unabhängige } \\
\text { Variablen }\end{array}$ & $\begin{array}{l}\text { Gesamt } \\
\text { marg. Eff } \\
\text { in \% }\end{array}$ & $\begin{array}{l}\text { Allein- } \\
\text { erziehend } \\
\text { marg. Eff. } \\
\text { in \% }\end{array}$ & $\begin{array}{l}\text { Im Paar- } \\
\text { Haushalt } \\
\text { marg. Eff. } \\
\text { in \% }\end{array}$ & $\begin{array}{l}\text { Gesamt } \\
\text { marg. Eff } \\
\text { in \% }\end{array}$ & $\begin{array}{l}\text { Allein- } \\
\text { erziehend } \\
\text { marg. Eff } \\
\text { in \% }\end{array}$ & $\begin{array}{l}\text { Im Paar- } \\
\text { Haushalt } \\
\text { marg. Eff } \\
\text { in \% }\end{array}$ & $\begin{array}{l}\text { Gesamt } \\
\text { marg. Eff } \\
\text { in \% }\end{array}$ & $\begin{array}{l}\text { Allein- } \\
\text { erziehend } \\
\text { marg. Eff } \\
\text { in \% }\end{array}$ & $\begin{array}{l}\text { Im Paar- } \\
\text { Haushalt } \\
\text { marg. Eff } \\
\text { in \% }\end{array}$ & $\begin{array}{l}\text { Mittel- } \\
\text { wert }\end{array}$ \\
\hline \multicolumn{11}{|l|}{ GRUPPE 1} \\
\hline $\begin{array}{l}\text { Frau } \\
\text { Berufl. Ausbildung } \\
\text { ( } \mathrm{R}^{\mathrm{a})} \text { : ohne/andere } \\
\text { Ausbildung/Lehre) }\end{array}$ & $9,2 * *$ & $11,6 * *$ & $8,8 * *$ & 0,3 & 0,4 & 0,2 & 0,0 & 0,0 & 0,0 & 0,54 \\
\hline - Studium & $-11,6 * * *$ & $-13,8 * * *$ & $-11,0 * * *$ & $-6,9 *$ & $-9,1 *$ & $-6,5 *$ & $-5,1$ & $-7,1$ & $-4,8$ & 0,25 \\
\hline $\begin{array}{l}\text { Alter jüngstes Kind } \\
\text { Partnerln im Haushalt } \\
\text { (R: nein) }\end{array}$ & 0,2 & 0,2 & 0,2 & $0,6 * *$ & $0,9 * *$ & $0,6 * *$ & $0,5 *$ & $0,8 *$ & $0,5^{*}$ & 8,51 \\
\hline $\begin{array}{l}\text { - Partnerln im Haushalt } \\
\text { Betreuungsnetzwerk } \\
\text { (R: kein) }\end{array}$ & $22,3 * * *$ & & & & & & & & & 0,89 \\
\hline- Groß & $-10,4 * * *$ & $-12,3 * * *$ & $-9,9 * * *$ & $-8,7 * *$ & $-11,3 * *$ & $-8,2 * *$ & $-8,1^{*}$ & $-10,8 * *$ & $-7,5 *$ & 0,19 \\
\hline $\begin{array}{l}\text { - Klein } \\
\text { Hauptverantwortung } \\
\text { Kinderbetreuung } \\
\text { (R: ich selbst) }\end{array}$ & 8,0 & $9,5 *$ & 7,6 & 2,6 & 3,5 & 2,5 & 2,2 & 3,1 & 2,0 & 0,86 \\
\hline - Partnerln & $-11,7 * *$ & $-14,1 * *$ & $-11,2 * *$ & $-6,5$ & $-8,6$ & $-6,1$ & $-7,0$ & $-9,6$ & $-6,5$ & 0,27 \\
\hline \multicolumn{11}{|l|}{ GRUPPE 2} \\
\hline \multicolumn{11}{|l|}{$\begin{array}{l}\text { Derzeitige Tätigkeit } \\
\text { (R: einf. Angestellte) }\end{array}$} \\
\hline $\begin{array}{l}\text { - qualif. Angestellte } \\
\text { - leitende Angestellte } \\
\text { - Arbeiterln }\end{array}$ & & & & $\begin{array}{c}-9,2 * \\
-1,2 \\
-11,6 *\end{array}$ & $\begin{array}{l}-11,9 * * \\
-1,6 \\
-14,2 *\end{array}$ & $\begin{array}{c}-8,6^{*} \\
-1,1 \\
-11,0^{*}\end{array}$ & $\begin{array}{l}-10,1 * * \\
-5,2 \\
-8,9\end{array}$ & $\begin{array}{l}-13,5 * * \\
-7,1 \\
-11,5\end{array}$ & $\begin{array}{l}-9,4^{* *} \\
-4,8 \\
-8,3\end{array}$ & $\begin{array}{l}0,25 \\
0,15 \\
0,07\end{array}$ \\
\hline $\begin{array}{l}\text { Tatsächliche Arbeitszeit/ } \\
\text { Woche (R: } 42 \text { Std. und mehr) } \\
\text { - 1-19,5 Std./Woche } \\
-20-29,5 \text { Std./Woche } \\
\text { - 30-39,5 Std./Woche } \\
\text { - 40-41,5 Std./Woche }\end{array}$ & & & & $\begin{array}{r}15,0 * * * \\
10,2 * * * \\
10,3 * * * \\
7,1 * *\end{array}$ & $\begin{array}{l}24,0 * * * \\
15,3 * * \\
15,3 * * * \\
10,4 * *\end{array}$ & $\begin{array}{r}13,9 * * * \\
9,5 * * * \\
9,5 * * * \\
6,6 * *\end{array}$ & $\begin{array}{c}12,7 * * * \\
10,0 * * * \\
10,8 * * * \\
6,2 *\end{array}$ & $\begin{array}{l}20,9 * * * \\
15,6 * * \\
17,0 * * * \\
9,4 *\end{array}$ & $\begin{array}{r}11,6 * * * \\
9,2 * * * \\
10,0 * * * \\
5,7 *\end{array}$ & $\begin{array}{l}0,11 \\
0,18 \\
0,19 \\
0,16\end{array}$ \\
\hline $\begin{array}{l}\text { Differenz tatsächl./gewünschte } \\
\text { Arbeitszeit pro Woche }\end{array}$ & & & & $-0,7 * * *$ & $-1,0 * * *$ & $-0,7 * * *$ & $-0,6 * * *$ & $-0,9 * * *$ & $-0,6 * * *$ & 8,18 \\
\hline $\begin{array}{l}\text { Abendarbeit } \\
\text { (R: selten, nie) } \\
\text { - immer, häufig }\end{array}$ & & & & $-6,8$ & $-8,8 *$ & $-6,4$ & $-7,4 *$ & $-10,0 *$ & $-6,9 *$ & 0,19 \\
\hline $\begin{array}{l}\text { Samstags- oder Sonntagsarbeit } \\
\text { (R: selten, nie) }\end{array}$ & & & & & 0,0 & & & & & \\
\hline $\begin{array}{l}\text { - immer, häufig } \\
\text { - gelegentlich }\end{array}$ & & & & $\begin{array}{l}-15,6 * * * \\
-11,7 * * *\end{array}$ & $\begin{array}{l}-19,2 * * * \\
-14,9 * * *\end{array}$ & $\begin{array}{l}-14,8 * * * \\
-11,0 * * *\end{array}$ & $\begin{array}{l}-14,9 * * * \\
-12,5 * * *\end{array}$ & $\begin{array}{l}-18,9 * * * \\
-16,5 * * *\end{array}$ & $\begin{array}{l}-13,9 * * * \\
-11,7 * * *\end{array}$ & $\begin{array}{l}0,21 \\
0,26\end{array}$ \\
\hline $\begin{array}{l}\text { Arbeit auf Abruf } \\
\text { (R: selten, nie) } \\
\text { - immer, häufig }\end{array}$ & & & & $-16,2 * *$ & $-18,9 * * *$ & $-15,4 * *$ & $-16,3 * *$ & $-19,5 * * *$ & $-15,3 * *$ & 0,07 \\
\hline $\begin{array}{l}\text { Dauer der AZ schwankt täglich } \\
\text { (R: selten, nie) } \\
\text { - gelegentlich }\end{array}$ & & & & $-8,7 * *$ & $-11,1 * *$ & $-8,2 * *$ & $-7,4^{*}$ & $-9,9 * *$ & $-6,9 *$ & 0,18 \\
\hline $\begin{array}{l}\text { Arbeitszeit zu } \\
\text { unvorhersehbaren Zeiten } \\
\text { (R: selten, nie) } \\
\text { - immer, häufig } \\
\text { - gelegentlich }\end{array}$ & & & & $\begin{array}{r}-12,1 * \\
-8,3 * *\end{array}$ & $\begin{array}{l}-14,7 * * \\
-10,7 * *\end{array}$ & $\begin{array}{r}-11,4 * \\
-7,8 * *\end{array}$ & $\begin{array}{r}-11,5 * \\
-9,1 * *\end{array}$ & $\begin{array}{l}-14,5 * \\
-12,1 * *\end{array}$ & $\begin{array}{l}-10,8 \\
-8,5 * *\end{array}$ & $\begin{array}{l}0,07 \\
0,17\end{array}$ \\
\hline \multicolumn{11}{|l|}{ GRUPPE 3} \\
\hline $\begin{array}{l}\text { Vorhandensein } \\
\text { Arbeitszeitmodelle (R: nein) } \\
\text { - anderes Arbeitszeitkonto } \\
\text { - Telearbeit möglich }\end{array}$ & & & & & & & $\begin{array}{rl}-13,2 & * * * \\
6,5 & * *\end{array}$ & $\begin{aligned}-16,5 * * * \\
9,7 * *\end{aligned}$ & $\begin{array}{r}-12,4 * * * \\
6,0 * *\end{array}$ & $\begin{array}{l}0,12 \\
0,23\end{array}$ \\
\hline $\begin{array}{l}\text { Bestimmung der } \\
\text { Arbeitsabläufe (R: selbst) } \\
\text { - Vorgesetzter }\end{array}$ & & & & & & & $-8,6 * *$ & $-11,7 * *$ & $-8,0 * *$ & 0,30 \\
\hline $\begin{array}{l}\text { Versäumte Arbeit } \\
\text { (R: durch andere vertreten) } \\
\text { - teils selbst nachholen, } \\
\text { teils vertreten }\end{array}$ & & & & & & & $-8,5 * *$ & $-11,5 * *$ & $-8,0 * *$ & 0,25 \\
\hline $\begin{array}{l}\text { Berücksichtigung } \\
\text { familiärer Belange } \\
\text { (R: eher nein/gar nicht) } \\
\text { - bei der Arbeitszeitfestlegung }\end{array}$ & & & & & & & $12,7 * * *$ & $16,8 * * *$ & $11,9 * * *$ & 0,74 \\
\hline $\begin{array}{l}\text { BIK Region } \\
\text { (R: über } 500.000 \text { Ew.) }\end{array}$ & & & & & & & & & & \\
\hline - 2.001 bis 5.000 Ew. & & & & & & & $11,1 * * *$ & $18,2 * * *$ & $10,2 * * *$ & 0,08 \\
\hline Gültige Fälle & 1.403 & & & 1.403 & & & 1.403 & & & \\
\hline McFaddens Pseudo $\mathrm{R}^{2}$ & $0,057 * * *$ & & & $0,192 * * *$ & & & $0,242 * * *$ & & & \\
\hline
\end{tabular}




\begin{tabular}{|c|c|c|c|c|c|}
\hline & Modell 1 & Modell 2 & Modell 3 & Modell 4 & \\
\hline Unabhängige Variablen & $\begin{array}{l}\text { Gesamt } \\
\text { marg. Eff. } \\
\text { in \% }\end{array}$ & $\begin{array}{l}\text { Gesamt } \\
\text { marg. Eff. } \\
\text { in \% }\end{array}$ & $\begin{array}{l}\text { Gesamt } \\
\text { marg. Eff. } \\
\text { in \% }\end{array}$ & $\begin{array}{l}\text { Gesamt } \\
\text { marg. Eff. } \\
\text { in \% }\end{array}$ & $\begin{array}{l}\text { Mittel- } \\
\text { wert }\end{array}$ \\
\hline \multicolumn{6}{|l|}{ GRUPPE 1} \\
\hline Frau & $11,6 * * *$ & 5,1 & 1,7 & 1,6 & 0,54 \\
\hline Alter jüngstes Kind & 0,5 & $0,8 * *$ & 0,6 & 0,5 & 8,50 \\
\hline \multicolumn{6}{|l|}{ GRUPPE 2} \\
\hline \multicolumn{6}{|l|}{$\begin{array}{l}\text { Derzeitige Tätigkeit } \\
\left(R^{a)} \text { : einfache Angestellte) }\right.\end{array}$} \\
\hline - mittlere Angestellte & & $-9,7 *$ & $-12,0 * *$ & $-10,5 *$ & 0,13 \\
\hline - qualif. Angestellte & & $-9,5 * *$ & $-12,0 * *$ & $-8,2$ & 0,25 \\
\hline \multicolumn{6}{|l|}{$\begin{array}{l}\text { Tatsächliche Arbeitszeit/Woche } \\
\text { (R: } 42 \text { Std. und mehr) }\end{array}$} \\
\hline - 1-19,5 Std./Woche & & $9,7 *$ & 6,7 & 4,9 & 0,11 \\
\hline - 20-29,5 Std./Woche & & $17,5 * * *$ & $17,6 * * *$ & $18,3 * * *$ & 0,18 \\
\hline - 30-39,5 Std./Woche & & $6,8 *$ & $8,5 * *$ & $8,6 *$ & 0,19 \\
\hline - 40-41,5 Std./Woche & & $7,4 *$ & 6,2 & 6,4 & 0,16 \\
\hline \multicolumn{6}{|l|}{$\begin{array}{l}\text { Nachtarbeit } \\
\text { (R: selten, nie) }\end{array}$} \\
\hline - immer, häufig & & 4,4 & 8,1 & $11,7 * *$ & 0,07 \\
\hline \multicolumn{6}{|l|}{$\begin{array}{l}\text { Samstags- oder Sonntagsarbeit } \\
\text { (R: selten, nie) }\end{array}$} \\
\hline - immer, häufig & & $-9,7 * *$ & $-7,4$ & $-9,4 *$ & 0,21 \\
\hline \multicolumn{6}{|l|}{$\begin{array}{l}\text { Dauer der AZ schwankt täglich } \\
\text { (R: selten, nie) }\end{array}$} \\
\hline - gelegentlich & & $-6,8 *$ & $-7,1 *$ & $-2,9$ & 0,19 \\
\hline \multicolumn{6}{|l|}{$\begin{array}{l}\text { Arbeit zu unvorhersehbaren Zeiten } \\
\text { (R: selten, nie) }\end{array}$} \\
\hline - gelegentlich & & $-10,0 * *$ & $-11,5 * *$ & $-10,7 * *$ & 0,17 \\
\hline \multicolumn{6}{|l|}{ GRUPPE 3} \\
\hline \multicolumn{6}{|l|}{$\begin{array}{l}\text { Vorhandensein Arbeitszeitmodelle } \\
\text { (R: nein) }\end{array}$} \\
\hline - Gleitzeitarbeit & & & $9,1 * * *$ & $8,0 * *$ & 0,45 \\
\hline - Telearbeit möglich & & & $13,0 * * *$ & $11,9 * * *$ & 0,23 \\
\hline \multicolumn{6}{|l|}{$\begin{array}{l}\text { Bestimmung der Arbeitsabläufe } \\
\text { (R: selbst) }\end{array}$} \\
\hline - Vorgesetzter & & & $-8,5 *$ & $-4,0$ & 0,30 \\
\hline \multicolumn{6}{|l|}{$\begin{array}{l}\text { Vorhandensein betriebliche soziale } \\
\text { Infrastruktur } \\
\text { (R: nein) }\end{array}$} \\
\hline - Facilities & & & $10,4 * * *$ & $10,2 * * *$ & 0,28 \\
\hline - monetäre Unterstützung & & & $11,7 * * *$ & $11,1 * * *$ & 0,64 \\
\hline \multicolumn{6}{|l|}{$\begin{array}{l}\text { Vorhandensein Notfallbetreuung } \\
\text { (R: nein) }\end{array}$} \\
\hline Notfallbetreuung & & & $8,9 * *$ & 6,1 & 0,23 \\
\hline \multicolumn{6}{|l|}{$\begin{array}{l}\text { Berücksichtigung familiärer Belange } \\
\text { (R: eher nein/gar nicht) }\end{array}$} \\
\hline - bei der Arbeitszeitfestlegung & & & $26,9 * * *$ & $23,7 * * *$ & 0,74 \\
\hline - bei der Urlaubsplanung & & & $14,6 * * *$ & 8,2 & 0,88 \\
\hline \multicolumn{6}{|l|}{$\begin{array}{l}\text { Beschäftigtenanzahl des Betriebes } \\
\text { (R: mehr als } 500 \text { Beschäftigte) }\end{array}$} \\
\hline - bis 5 Beschäftigte & & & $19,0 * * *$ & $17,6 * * *$ & 0,18 \\
\hline - 6 bis 15 Beschäftigte & & & $9,2 *$ & 5,0 & 0,21 \\
\hline \multicolumn{6}{|l|}{$\begin{array}{l}\text { BIK Region } \\
\text { (R: über } 500.000 \mathrm{Ew} .)\end{array}$} \\
\hline - 2.001 bis $5.000 \mathrm{Ew}$ & & & 7,7 & $10,4 * *$ & 0,09 \\
\hline \multicolumn{6}{|l|}{ GRUPPE 4} \\
\hline \multicolumn{6}{|l|}{$\begin{array}{l}\text { Reaktion KollegInnen positiv (R: } \\
\text { neutral/noch nicht vorgekommen) }\end{array}$} \\
\hline - Fehlzeit, weil Kind krank & & & & $19,9 * * *$ & 0,24 \\
\hline - Keine Überstunden wg. Kind & & & & $10,1 *$ & 0,16 \\
\hline - Keine Wochenendarb. wg. Kind & & & & $-13,3 *$ & 0,11 \\
\hline \multicolumn{6}{|l|}{$\begin{array}{l}\text { Reaktion KollegInnen negativ (R: } \\
\text { neutral, noch nicht vorgekommen) }\end{array}$} \\
\hline - Fehlzeit, weil Kind krank & & & & $-21,5 * * *$ & 0,12 \\
\hline - Keine Überstunden wg. Kind & & & & $-12,4 * *$ & 0,18 \\
\hline Gültige Fälle & 1.354 & 1.354 & 1.354 & 1.354 & \\
\hline McFaddens Pseudo $\mathrm{R}^{2}$ & $0,019 * * *$ & $0,067 * * *$ & $0,194 * * *$ & $0,258 * * *$ & \\
\hline
\end{tabular}

Einwohnern leben, haben eine deutlich höhere Wahrscheinlichkeit, die Vereinbarkeit von Arbeitszeit und Familie als gut einzuschätzen als Großstadtbewohner. Das könnte auf Zeiten für (Arbeits-)Wege und eine erschwerte Nutzung familiärer oder nachbarschaftlicher Netzwerke zurückzuführen sein.

\section{GUTE BEWERTUNG DER FAMILIEN- FREUNDLICHKEIT DES BETRIEBES (BTGUT)}

Die signifikanten marginalen Effekte der logistischen Regression auf die gute Beurteilung der Familienfreundlichkeit des Betriebes (BTGUT) gehen aus Tabelle 2 hervor.

Im ersten Modell zeigt sich, dass ausschließlich das Geschlecht signifikanten Einfluss auf die gute Bewertung der Familienfreundlichkeit des Betriebes hat: Frauen haben eine höhere Wahrscheinlichkeit, dem Betrieb eine gute Familienfreundlichkeit zu bescheinigen, als Männer. Dieser Effekt verschwindet jedoch unter Berücksichtigung beruflicher und betrieblicher Merkmale.

Womit können die Betriebe aus Sicht der Arbeitnehmerinnen und Arbeitnehmer am stärksten die Familienfreundlichkeit erhöhen? Wenn bei der Arbeitszeitfestlegung die familiären Belange berücksichtigt werden, hat das den höchsten positiven Effekt auf die Wahrscheinlichkeit, dass der Betrieb als familienfreundlich bewertet wird.

Signifikant ist die Dauer der tatsächlichen Arbeitszeit: Für die Bewertung der Familienfreundlichkeit ist die Möglichkeit wichtig, Teilzeit in einem substanziellen Stundenbereich zu arbeiten. Frauen und Männer mit einer tatsächlichen Arbeitszeit zwischen 20 bis 29,5 Stunden pro Woche haben eine deutlich höhere Wahrscheinlichkeit einer guten Bewertung der Familienfreundlichkeit des Betriebes als diejenigen, die länger als 42 Stunden arbeiten.

Auch ein familienfreundliches betriebliches Klima erhöht die Wahrscheinlichkeit einer guten Bewertung des Betriebes. Bemerkenswert ist die weitere Steigerung der Modellanpassung von Modell 3 zu Modell 4, bei welchem Reaktionen von Kollegen auf vereinbarkeitsbedingte Fehlzeiten als zusätzliche Variablen berücksichtigt werden. Diese Merkmale des Betriebsklimas steigern die Erklärungskraft des Modells 
und sind einflussreicher als viele andere Variablen. Mütter und Väter bewerten ihren Betrieb offenbar in starkem Maße danach, ob ihnen die Vereinbarkeit durch verständnisvolle Reaktionen ihrer Kolleginnen und Kollegen erleichtert wird oder nicht. Positive Reaktionen auf Fehlzeiten wegen eines kranken Kindes gehen mit einer $20 \%$ höheren Wahrscheinlichkeit der guten Bewertung der Familienfreundlichkeit einher - im Vergleich zu neutralen Reaktionen. Der umgekehrte Zusammenhang gilt ebenso: Wird im Betrieb negativ auf Fehlzeiten wegen eines kranken Kindes reagiert, verringert dies die Wahrscheinlichkeit einer guten Bewertung der Familienfreundlichkeit deutlich.

Daneben ist Telearbeit ein positiv wirkender Faktor. Auch familienfreundliche Angebote („Facilities“), wie z. B. Kinderspielzimmer im Betrieb oder Freizeitangebote für Kinder ebenso wie monetäre familienbezogene Maßnahmen, werden geschätzt. Der signifikant positive Einfluss einer Notfallbetreuung für Kinder besteht nur im dritten Modell, bleibt jedoch unter Berücksichtigung des Betriebsklimas im Modell 4 nicht mehr erhalten. Insgesamt wirken sich die beiden ersten Faktoren Berücksichtigung bei der Arbeitszeitfestlegung und das Verständnis der Kolleginnen und Kollegen - viel stärker aus.

Überraschenderweise wirkt Nachtarbeit positiv auf die Bewertung der Familienfreundlichkeit des Betriebes. Offenbar wissen die Beschäftigten bei der Wahl eines Arbeitsplatzes, worauf sie sich einlassen, und nutzen Nachtarbeit unter den gegebenen Bedingungen als Vereinbarkeitsstrategie.

Bemerkenswert ist die Bedeutung der Betriebsgröße: In einem kleinen Betrieb mit bis zu fünf Beschäftigen tätig zu sein, erhöht die Wahrscheinlichkeit der guten Bewertung der Familienfreundlichkeit deutlich im Vergleich zur Beschäftigung in einem Betrieb mit mehr als 500 Beschäftigten.

\section{Zusammenfassung und Schlussfolgerungen}

Durch die multivariate Analyse konnte ermittelt werden, welche der bivariaten $\mathrm{Zu}$ sammenhänge unter Berücksichtigung aller anderen Variablen bestehen bleiben und wie stark die Effekte sind. Dabei hat sich die Lebensform als gewichtiger Einflussfaktor für die Vereinbarkeit von Arbeitszeit und Familie herausgestellt: Alleinerziehende haben eine deutlich niedrigere Wahrscheinlichkeit als Eltern in Paarhaushalten, die Vereinbarkeit von Arbeitszeiten und Familie gut zu bewerten. Auf die Bewertung der Familienfreundlichkeit wirkt besonders positiv, wenn familiäre Belange bei der Arbeitszeitfestlegung berücksichtigt werden. An zweiter Stelle für die Familienfreundlichkeit steht das Betriebsklima.

Die multivariate Analyse verdeutlicht die große Bedeutung der Dauer der Arbeitszeit. Deutlich positiv wirken sich kurze tatsächliche Arbeitszeiten aus; deutlich negativ Arbeit am Wochenende. Eine Lage der Arbeitszeit zuzeiten, in denen die Versorgung der Kinder notwendig ist und typischerweise gemeinsame Familienzeiten gelebt werden, führt zu einer negativen Bewertung von Vereinbarkeit. Gleiches gilt für Arbeit auf Abruf.

Die Bedeutung flexibler Arbeitszeitmodelle für die Vereinbarkeit von Familie und Beruf wird durch die Auswertung stark relativiert. Obwohl man annehmen könnte, dass sie eine familienbezogene Flexibilität erleichtern, führen Überstundenkonten, Arbeitszeitabstimmung im Team ebenso wie Vertrauensarbeitzeiten nicht zu signifikant höherer Wahrscheinlichkeit einer guten Bewertung der Vereinbarkeit und der Familienfreundlichkeit des Betriebes. Einzig Gleitzeitarbeit erhöht die Wahrscheinlichkeit, dass der Betrieb als familienfreundlich eingeschätzt wird. Andere Arbeitszeitkonten dagegen scheinen vor allem der betrieblich gesteuerten Flexibilisierung der Arbeitszeiten zu dienen. Ihr Vorhandensein hat signifikant negativen Einfluss auf die Vereinbarkeitsbewertung.

Überraschen könnte auf den ersten Blick, dass spezielle familienfreundliche Maßnahmen sich nicht so stark auswirken wie andere betriebliche Bedingungen. Ein familienfreundliches Betriebsklima mit unterstützenden Kollegenreaktionen ist von stärkerem Gewicht, ebenso die Berücksichtigung familiärer Belange bei der Arbeitszeitfestlegung. Dass die familienfreundlichen Maßnahmen keinen stärkeren Effekt haben, kann daran liegen, dass sie oftmals auf spezielle Situationen (z. B. Vermittlung eines Betreuungsplatzes, Notfallbetreuung) zugeschnitten sind und damit nur selten genutzt werden, wohingegen das Betriebsklima alltäglich relevant ist.

Im Widerspruch zu den Annahmen und nicht leicht zu interpretieren sind die Ergebnisse, dass Drei-Schichtarbeit keinen signifikanten Einfluss hat und auch Nachtarbeit bei keiner der abhängigen Variablen signifikant negative Effekte auf die Wahrscheinlichkeit einer guten Bewertung zeigt. Beide Arbeitszeitformen könnten als Strategien der Vereinbarkeit genutzt werden, ungeachtet möglicher negativer Folgen. Mehr Augenmerk sollte künftig auf die Gründe dafür gerichtet werden, dass die Kleinstbetriebe als so viel familienfreundlicher eingeschätzt werden.

Unter den außerbetrieblichen Bedingungen tritt vor allem hervor, dass ein Wohnumfeld von nicht mehr als 2.000 bis 5.000 Einwohnern die Wahrscheinlichkeit einer guten Bewertung der Vereinbarkeit von Arbeitszeit und Familie wie auch der guten Bewertung der Familienfreundlichkeit des Betriebes stark positiv beeinflusst. Künftige Forschung sollte diesen Befunden nachgehen.

Für betriebliches Handeln ergeben sich folgende Schlussfolgerungen: Um Betriebe familienfreundlicher zu machen, kommt es weniger darauf an, neue "moderne“ flexible Arbeitszeitmodelle $\mathrm{zu}$ installieren oder spezielle familienfreundliche Maßnahmen einzuführen - obwohl das durchaus im Einzelfall hilfreich sein kann. Wichtiger sind seit Langem bekannte „traditionelle" Handlungsfelder: die Möglichkeit der Teilzeitarbeit oberhalb von 20 Stunden, die Begrenzung der Arbeitszeitdauer (Vermeidung von Überstunden) und die Minimierung von familienfeindlichen Arbeitszeiten wie Arbeit am Wochenende und am Abend. Müttern und Vätern ist vor allem wichtig, dass ihre Belange bei der Arbeitszeitfestlegung im Betrieb berücksichtigt werden. Wenn sie aber bestimmte Arbeitszeiten nicht übernehmen können oder wegen der Kinder fehlen müssen, wollen sie, dass die Vorgesetzten und Kollegen die Inanspruchnahme von familienfreundlichen Maßnahmen vollständig akzeptieren und es ohne negative Konsequenzen für die berufliche Karriere bleibt, wenn sie Freistellungsrechte in Anspruch nehmen. Das Betriebsklima, gemessen am Verständnis und den Reaktionen von Kolleginnen und Kollegen, ist ihnen wichtiger als diese oder jene spezielle Maßnahme.

Unter den konkreten Maßnahmen sind es vor allem Gleitzeitarbeit sowie Tele- 
arbeit und die Möglichkeit der Notfallbetreuung für Kinder, die sich positiv auf die Einschätzungen seitens der Eltern auswirken.

Das verweist darauf, dass es für mehr Familienfreundlichkeit nicht in allererster
Linie um aufwendige Investitionen in eine Infrastruktur familienfreundlicher Maßnahmen geht, sondern um einen anderen Umgang mit Beschäftigten, die Familienaufgaben haben. Arbeitszeit und Arbeitsorganisation entsprechend zu gestalten und ein aufgeschlossenes Klima im Betrieb gegenüber den Bedürfnissen von Erziehenden - das macht Betriebe familienfreundlich.

\section{ITERATUR}

Bauer, F./Groß, H./Lehmann, K./Munz, E. (2004): Arbeitszeit 2003. Arbeitszeitgestaltung, Arbeitsorganisation und Tätigkeitsprofile, Köln Bloom, N./Kretschmer, T./van Reenen, J. (2006): Work-Life-Balance, Management Practices and Productivity, Center for Economic Performance, LSE London

BMFSFJ (2004): Erwartungen an einen familienfreundlichen Betrieb. Erste Auswertung einer repräsentativen Befragung von Arbeitnehmerinnen und Arbeitnehmern mit Kindern oder Pflegeaufgaben, Berlin

BMFSFJ/Bundesverband der deutschen Industrie (BDI)/Institut der deutschen Wirtschaft Köln (IW) (2004): Bevölkerungsorientierte Familienpolitik - ein Wachstumsfaktor, Berlin/Köln BMFSFJ/Institut der deutschen Wirtschaft Köln (IW) 2005: Familienfreundliche Regelungen in Tarifverträgen und Betriebsvereinbarungen. Beispiele guter Praxis. http://www.bmfsfj.de/Politikbereiche/Familie/ familie-und-arbeitswelt,did=11408.html. (10.Mai 2007)

Bond, J. T./Thompson, C./Galinski, E./Prottas, D. (2003): Highlights of the National Study of the changing workforce, Families and Work Institute 3

Bundesmann-Jansen, J./Groß, H./Munz, E. (2000): Arbeitszeit '99, hrsg. vom Ministerium für Arbeit, Soziales und Stadtentwicklung, Kultur und Sport des Landes NRW, Köln

Büssing, A./Seifert, H. (1995): Sozialverträgliche Arbeitszeitgestaltung, München und Mering

Dilger, A./Gerlach, I./Schneider, H. (Hrsg.) (2007): Betriebliche Familienpolitik. Potenziale und Instrumente aus multidisziplinärer Sicht, Wiesbaden

Eberling, M./Hielscher, V./Hildebrandt, E./Jürgens, K. (2004): Prekäre Balancen. Flexible Arbeitszeiten zwischen betrieblicher Regulierung und individuellen Ansprüchen, Berlin

European Foundation for the Improvement of Living and Working Conditions (2003): Working time preferences and work-life balance in the EU: some policy considerations for enhancing the quality of life, Dublin Flüther-Hoffmann, C./Seyda, S. (2006): Unternehmensmonitor Familienfreundlichkeit 2006. Wie familienfreundlich ist die deutsche Wirtschaft?, hrsg. vom BMFSFJ und IW Köln

Flüther-Hoffmann, C./Solbrig, J. (2003): Wie familienfreundlich ist die deutsche Wirtschaft? in: IW-Trends 4, S. 1-18

Habisch, A. (Hrsg.) (1995): Familienorientierte Unternehmensstrategie. Beiträge zu einem zukunftsorientierten Programm, München und Mehring

Juncke, D. (2005): Betriebswirtschaftliche Effekte familienbewusster Personalpolitik: Forschungsstand, in: Forschungszentrum Familienbewusste Personalpolitik an der Westfälischen Wilhelms-Universität, Arbeitspapier 1
Klenner, C./Pfahl, S. (2005): Stabilität und Flexibilität - Ungleichmäßige Arbeitszeitmuster und familiale Arrangements, in: Seifert, H. (Hrsg): Flexible Zeiten in der Arbeitswelt, Frankfurt/New York, S. 124-169 La Valle, I./Arthur, S./Millward, Ch./Scott, J./Clayden, M. (2002): Happy families? Atypical work and its influence on family life, Bristol Long, S./Freese, J. (2006): Regression Models for Categorical Dependent Variables Using Stata, College Station

Maschke, M./Pfahl, S./Reuyß, S. (2005): Familienfreundlichkeit im Betrieb - Handlungshilfe für die betriebliche Interessenvertretung, hrsg. vom BMFSFJ Berlin

Möller, I./Allmendinger, J. (2003): Frauenförderung: Betriebe könnten noch mehr für die Chancengleichheit tun, IAB-Kurzbericht 12 vom 6.8. Munz, E. (2006): Mehr Balance durch selbst gesteuerte Arbeitszeiten?, in: WSI-Mitteilungen 9, S. 478-484

OECD (2001): Employment Outlook, Paris

OECD (2004): Recent Labour Market Developments and Prospects, Employment Outlook, Paris

Prognos (2003): Betriebswirtschaftliche Effekte familienfreundlicher Maßnahmen - Kosten-Nutzen-Analyse, Berlin/Basel

Prognos (2004): Familienfreundliche Maßnahmen im Handwerk. Potenziale, Kosten-Nutzen-Relationen, Best Practices, Berlin/Basel

Richter, G./Stackelbeck, M. (1992): Beruf und Familie. Arbeitszeitpolitik für Eltern kleiner Kinder, Köln

Rinderspacher, J. P. (1987): Am Ende der Woche. Die soziale und kulturelle Bedeutung des Wochenendes, Bonn

Rürup, B./Gruescu, S. (2003): Nachhaltige Familienpolitik im Interesse einer aktiven Bevölkerungsentwicklung, Berlin

Sachverständigenkommission (2005): Siebter Familienbericht - Familie zwischen Flexibilität und Verlässlichkeit, im Auftrag des BMFSFJ

Saltzstein, A. L./Ting, Y./Saltzstein, G. H. (2001): Work-Family Balance and Job-satisfaction: The Impact of family-friendly Policies on Attitudes of Federal Government Employees, in: Public Administration Review, July/August 4, S. 452-467

Seifert, H. (Hrsg) (2005): Flexible Zeiten in der Arbeitswelt, Frankfurt/ New York

Thomas, L. T./Ganster, D. C. (1995): Impact of Family-Supportive Work Variables on Work-Family Conflict and Strain: A Control Perspektive, in: Journal of Applied Psychology 1, S. 6-15

Weber, D. (2006): Work-Family Balance: The Effects of Organizational Initiatives on Creating a Family-Supportive Work Environment, in: IAWReport I, S. 103-154 